\title{
THE PARALLAXES OF U GEM VARIABLES
}

Karl W. Kamper

David Dunlap Observatory

University of Toronto

An Allegheny parallax series of SS Cyg, consisting of 52 exposures obtained on 15 nights, was recently measured on the PDS microphotometer at the David Dunlap Observatory, and a value of +0":022 \pm 0.006 (m.e.) derived for the absolute parallax. This is close to the mean of the two previous discordant measures for this star given in the table below. The welghted mean of the three determinations implies that the absolute magnitude, at quiescent phase, of the star is between 7.0 and 9.0 formally at a $90 \%$ confidence level. Recent parallax determinations made at Lick by Vasilevskis et al. (1975) for three other stars, 11sted below along with the Mt. Wilson value for $U$ Gem, imply even falnter absolute magnitudes.

\begin{tabular}{|c|c|c|c|c|c|c|}
\hline ss & Cyg & 0:019ะ0":005 & $M=8.2$ & from & $\begin{array}{r}-0.012 \pm 0.012 \\
0.032 \pm 0.010 \\
0.022 \pm 0.006\end{array}$ & $\begin{array}{l}\text { (Mt. Wilson) } \\
\text { (Yerkes) } \\
\text { (Allegheny) }\end{array}$ \\
\hline$n$ & Gem & $0.007 \pm 0.004$ & $M=8.6$ & from & $\begin{array}{l}0.012 \pm 0.010 \\
0.006 \pm 0.005\end{array}$ & $\begin{array}{l}\text { (Mt. Wilson) } \\
\text { (Lick) }\end{array}$ \\
\hline SS & Aur & $0.010 \pm 0.004$ & $M=9.8$ & & & (LIck) \\
\hline & on & $0.015 \pm 0.006$ & $M=11.5$ & & & (Iflck) \\
\hline
\end{tabular}

Individually, all of these determinations are quite weak due to the small values involved but,collectively, they imply that the $U$ Gem stars are at least as faint as the value $M_{v}=+7.5$ found by Kraft and Luyten (1965) from a secular parallax solution.

We would like to thank Dr. G. Gatewood for the loan of the Allegheny Observatory plates.

\section{REFERENCES}

Kraft, R.P. and Luyten, W.J. 1965, Astrophys. J., 142, 1041.

Vasilevskis, S., Harlan, E.A., Klemola, A.R., and Wirtanen, C.A. 1975, Publ. Lick Obs., 23, part V 